\title{
INFLUENCE OF MECHANICAL STRESS ON THE BIREFRINGENCE PROPERTIES OF $\left(\mathrm{N}\left(\mathrm{CH}_{3}\right)_{4}\right)_{2} \mathrm{ZnCl}_{4}$ CRYSTALS
}

\author{
I. Polovinko, S. Sveleba, O. Vlokh and V. Zhmurko \\ Lviv University, Physical Department, Lomonosova str. 8, 290005, Lviv, Ukraine
}

(Received September 27, 1991; in final form January 2, 1992)

The influence of applied mechanical stress on the birefringence of $\left(\mathrm{N}\left(\mathrm{CH}_{3}\right)_{4}\right)_{2} \mathrm{ZnCl}_{4}$ crystal in the temperature region of its incommensurate phase was investigated using Senarmonth's method $(\lambda=633 \mathrm{~nm})$. The value of pinning force for the samples with different defectiveness was experimentally determined.

PACS numbers: $77.80 . \mathrm{Bh}$

As it is known, the interaction of incommensurate (IC) structure with defects causes some essential properties of IC-phases. The temperature hysteresis of IC modulation wave vector [1], dielectric permittivity [2] and linear birefringence [3] may be related to such properties. The occurrence of temperature hysteresis is an evidence of the presence of some mechanisms preventing the processes of solitons creation and annihilation [1,2]. One of such mechanisms may be soliton pinning on impurities, vacancies, dislocations and so on. Experimental studies of impure crystals confirm the above-mentioned mechanism of temperature hysteresis [4]. Irregular character of the pinning force acting on solitons breaks a regular distribution of solitons in the vicinity of $T_{c}$, and brings the solitons to metastable chaotic state. Apart from the pinning force $\boldsymbol{F}_{\text {pin }}$, the solitons are influenced by the elastic coupling force $\boldsymbol{F}_{\text {el }}$ acting between solitons and, in the case of a crystal sample in an external field, by the applied force $\boldsymbol{F}_{\text {appl }}$. Therefore, the total force $\boldsymbol{F}=\boldsymbol{F}_{\mathrm{pin}}+\boldsymbol{F}_{\mathrm{el}}+\boldsymbol{F}_{\text {appl }}$ should be irregularly distributed in the sample volume.

The aim of this paper is to study the influence of the applied mechanical stresses on soliton-defect interaction and an experimental determination of the pinning force $\boldsymbol{F}_{\text {pin }}$ value. Ferroelectric $\left(\mathrm{N}_{(}\left(\mathrm{CH}_{3}\right)_{4}\right)_{2} \mathrm{ZnCl}_{4}$ crystals were chosen as the objects of our investigation, since a smooth variation of IC-parameter $\delta$ is characteristic of the IC-phase of these crystals at the temperatures $T_{\mathrm{c}}=280 \mathrm{~K}$ $\div T_{\mathrm{i}}=296.6 \mathrm{~K}$. The samples were grown using a slow evaporation method at room temperature from aqueous solution of $\mathrm{ZnCl}_{2}$ and $\mathrm{N}\left(\mathrm{CH}_{3}\right)_{4} \mathrm{Cl}$ salts taken in 
stoichiometric ratio. The influence of mechanical stresses on soliton-defects interaction was studied from the measurements of the linear birefringence increment dependences by Senarmonth's method $(\lambda=633 \mathrm{~nm})$. The axial mechanical stress was applied along the $a$ axis, since the spontaneous polarization $P_{a}$ in the ferroelectric phase is suppressed by $\sigma_{a}\left(T_{1}=276.3 \mathrm{~K} \div T_{c}=280 \mathrm{~K}\right)$ [5]. The light propagates along the modulation axis $c$.

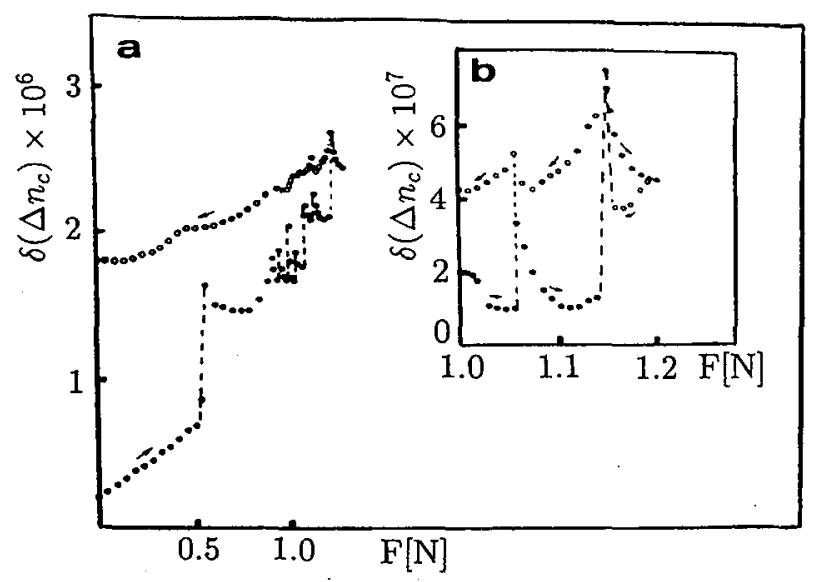

Fig. 1. The dependence of birefringence increment for the $c$-plate of $\left(\mathrm{N}\left(\mathrm{CH}_{3}\right)_{4}\right)_{2} \mathrm{ZnCl}_{4}$ sample on the value of $F_{a}$ applied along the $a$ axis at $T=288 \mathrm{~K}\left(\bullet-F_{a}\right.$ increasing regime, $\circ-F_{a}$ decreasing regime) (a) and the parts of corresponding curves in enlarged scale (b).

The experimental results are presented in Figs. 1-3. As it is shown in Fig. 1 for non-irradiated sample the $\delta(\Delta n)_{c}$ value increases at first. Then the $\delta(\Delta n)_{c}=$ $f(F)$ dependence is characterized by a sequence of peaks which are similar to the ones in the temperature dependence $\delta(\Delta n)_{\mathrm{i}}=f(T)$ at a low rate of temperature changes $(\partial T / \partial t=60 \mathrm{mK} / \mathrm{h})$ [6]. The so-called "viscous" interaction manifests itself if the velocities of the IC-structure motion and defect diffusion become close and the optimum conditions for soliton-defect interaction arise [6]. The force-velocity dependence describing soliton motion [7] acquires a hysteresis character at "viscous" interaction.

According to the data of Ref. [8], the applied mechanical stress changes the soliton density $x_{0} / d_{0}$, where $x_{0}$ is the intersoliton distance, $d_{0}$ - the soliton width. It leads to changes in the soliton-soliton interaction energy $E_{\mathrm{ss}}=a \exp \left(-\pi x_{0} / d_{0}\right)$ and the soliton-defect interaction energy $U_{\mathrm{b}}=n_{\mathrm{d}} x_{0} E_{\mathrm{b}}$ [9], where $n_{\mathrm{d}}$ denotes the defect concentration, $E_{\mathrm{b}}$ - the deféct contact energy. In the presence of defects the soliton pinning will be observed at the condition $n_{\mathrm{d}} x_{0} E_{\mathrm{b}} \approx a \exp \left(-\pi x_{0} / d_{0}\right)$. Therefore, the application and the release of mechanical stress will be accompanied by a hysteresis of $\delta(\Delta n)_{\mathrm{c}}$ value and $\delta(\Delta n)_{\mathrm{c}}=f(F)$ peaks positions. Hysteresis increases and peaks assymetry becomes more pronounced with increasing defectiveness (Figs. 1b, 2b, 3b). 

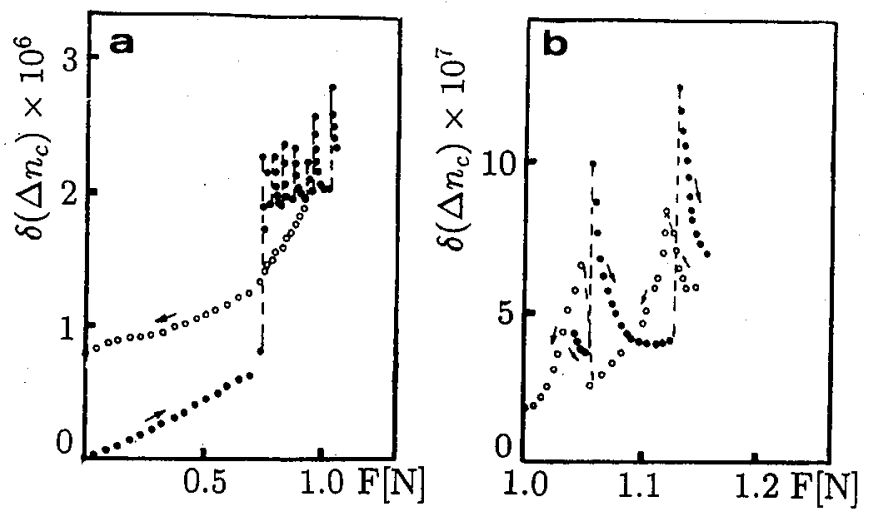

Fig. 2. The birefringence increment $\delta\left(\Delta n_{c}\right)$ dependence on $F_{a}$ value at $T=288 \mathrm{~K}$ for

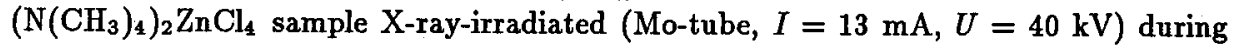
2 hours $\left(\bullet-F_{a}\right.$ increasing regime, $\circ-F_{a}$ decreasing regime) (a) and the parts of corresponding curves in enlarged scale (b).

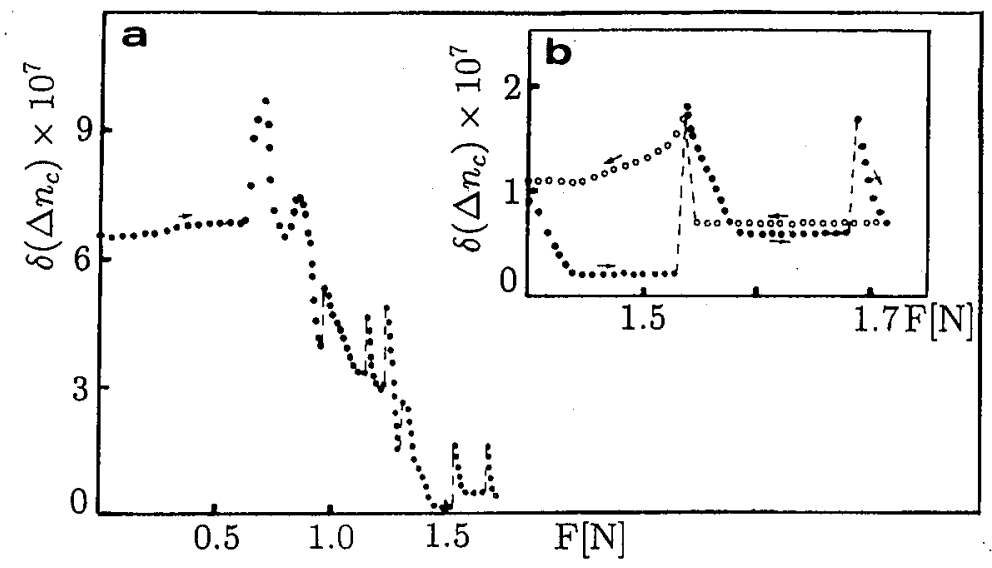

Fig. 3. The birefringence increment $\delta\left(\Delta n_{\mathrm{c}}\right)$ dependence on $F_{a}$ value ( $F_{a}$ increasing regime) at $T=288 \mathrm{~K}$ for $\left(\mathrm{N}\left(\mathrm{CH}_{3}\right)_{4}\right)_{2} \mathrm{ZnCl}_{4}$ sample in which the exponential distribution of defects due to X-ray-irradiation is created along the $c$ axis (a) and the $\sigma\left(\Delta n_{c}\right)$

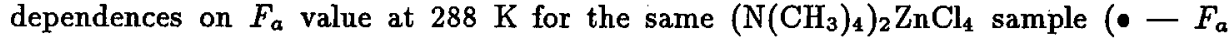
increasing regime, $0-F_{a}$ decreasing regime) in enlarged scale (b).

On the basis of the above result the pinning force value may be estimated as $F_{\text {pin }}=F_{\mathrm{k}} / n$, where $n$ is the soliton density, $F_{\mathrm{k}}$ - the coercitive force. The $2 F_{\mathrm{k}}$ value is determined as the difference between the $F_{\text {appl }}$ values for the corresponding peaks obtained on application and release of external stress (see Figs. 1b, 2b, 3b). The results $F_{\text {pin }}=1.2 \times 10^{-8}, 4 \times 10^{-8}, 21 \times 10^{-8} \mathrm{~N}$ are obtained for the Figs. $1 b, 2 b, 3 b$, respectively. It is obvious that an increase in the sample defectiveness 
increases the value of the pinning force. The values $F_{\text {pin }}$ found in this paper are in agreement with those obtained in [7] equal to $10^{-8} \mathrm{~N}$ for $\mathrm{Ba}_{2} \mathrm{Na} \cdot \mathrm{Nb}_{5} \mathrm{O}_{15}$ crystals.

Thus, an axial mechanical stress $\sigma_{a}$ leads to changes in the soliton density and, therefore, to changes in the conditions of soliton-defect interaction. The results obtained in this paper illustrate a set of hysteresis force-velocity dependences for description of soliton motion under mechanical field influence. Numerical values of $F_{\text {pin }}$ are in agreement with the theoretical estimation [7]. We suppose analogous behaviour for other IC crystals.

\section{References}

[1] K. Deguchi, Y. Okada, H. Fukunaga, E. Nakamura, J. Phys. Soc. Jpn. 56, 208 (1987).

[2] B.A. Strukov, V.M. Arutyunova, I. Uesu, Fiz. Tverd. Tela 24, 3061 (1982).

[3] O.G. Vlokh, I.I. Polovinko, V.I. Mokry, S.A. Sveleba, Ukr. Fiz. Zh. 35, 349 (1990).

[4] V.V. Lemanov, B. Brżezina, S.H. Esayan, A. Karaev, Fiz. Tverd. Tela 26, 1331 (1984).

[5] V.V. Gladkii, S.N. Kallaev, V.A. Kirikov et al., Abstracts of First Soviet-Poland Symp. of Ferroelectrics Phys., Lviv 1990, p. 221.

[6] O.G. Vlokh, V.S. Zhmurko, I.I. Polovinko, S.A. Sveleba, Ukr. Fiz. Zh. 35, 1493. (1990).

[7] D.J. Srolovitz, R. Eykholt, D.M. Barnett, J.P. Hirth, Phys. Rev. B 35, 6107 (1987).

[8] A.M. Dzhabrailov, V.A. Kirikov, V.V. Gladkii, I.S. Zheludev, Fiz. Tverd. Tela 27, 3465 (1985).

[9] P. Prelovsek, Ferroelectrics 54, 29 (1984). 